\title{
Características Biométricas del Cuerpo Calloso en Individuos chilenos
}

\author{
Biometric Characteristics of the Corpus Callosum in Chilean Subjects
}

"Olave, E.; *Torrez, J. C.; *** Riquelme, N.; ${ }^{* *}$ Ibacache, L. \& ${ }^{* * *}$ Binvignat, O.

OLAVE, E.; TORREZ, J. C.; RIQUELME, N. ; IBACACHE, L. \& BINVIGNAT, O. Características biométricas del cuerpo calloso en individuos chilenos. Int. J. Morphol., 30(4):1449-1452, 2012.

RESUMEN: El cuerpo calloso conecta ambos hemisferios cerebrales y se ubica en el fondo de la fisura longitudinal del cerebro. Con el propósito de complementar el conocimiento sobre esta importante estructura del sistema nervioso en nuestra población, determinamos diversos parámetros biométricos, que incluyeron su localización respecto a los márgenes anterior y posterior de los hemisferios cerebrales, longitud, espesor a diversos niveles, entre otras variables. Se utilizaron 20 cerebros frescos, de individuos adultos, a los cuales se fotografió la cara medial de sus hemisferios, los que fueron acompañados con una regla antes de obtener la fotografía, con el objetivo de trabajar sobre la imagen y, de este modo, registrar las variables planificadas. Estas medidas fueron registradas con un caliper digital de $0,05 \mathrm{~mm}$ de precisión. La distancia promedio obtenida entre la parte más prominente de la rodilla del cuerpo calloso y el margen anterior del hemisferio cerebral fue de $35,4 \mathrm{~mm}$ y, entre la parte más prominente del esplenio y el margen posterior del hemisferio cerebral fue de $52,8 \mathrm{~mm}$. La longitud antero-posterior del cuerpo calloso fue, en promedio, $88,2 \mathrm{~mm}$. La distancia antero-posterior promedio de la parte media de la rodilla fue $13,2 \mathrm{~mm}$ y la de la parte media del esplenio $15,7 \mathrm{~mm}$. Los datos obtenidos son un aporte al conocimiento biométrico y morfológico de esta importante estructura comisural, en nuestro grupo poblacional.

PALABRAS CLAVE: Anatomía; Sistema nervioso; Comisuras; Cuerpo calloso; Biometría.

\section{INTRODUCCIÓN}

El cuerpo calloso es una estructura de sustancia blanca que conecta ambos hemisferios cerebrales y se ubica en el fondo de la fisura longitudinal del cerebro. Se le considera un extremo anterior denominado pico o rostrum, que es la parte delgada; una rodilla, que es su extremo anterior curvo, que se dobla inferiormente por delante del septo pelúcido; un cuerpo, que se arquea posteriormente y termina como una porción posterior engrosada denominada esplenio. En su trayectoria lateral, las fibras de la rodilla se curvan hacia adelante en los lóbulos frontales y forman el fórceps menor (radiación frontal); las fibras del tronco se extienden lateralmente como la radiación del cuerpo calloso y las fibras en el esplenio se arquean hacia atrás en dirección al lóbulo occipital y forman el fórceps mayor o radiación occipital (Snell, 2001).

El cuerpo calloso forma un arco de aproximadamente $10 \mathrm{~cm}$ de longitud y su extremidad anterior se encuentra aproximadamente a $4 \mathrm{~cm}$ de los polos frontales (Williams et al., 1995). Diversos autores han informado sobre las característi- cas morfológicas de esta estructura, obtenidas de cerebros frescos o formolizados, así como también estudios imagenológicos en individuos vivos (Allen et al., 1991; Bishop \& Wahlsten, 1997; Dorion et al., 2001; Battal et al., 2010).

Con el propósito de complementar el conocimiento sobre esta importante estructura comisural en nuestra población, determinamos diversos parámetros entre los cuales destacamos su longitud, su espesor en diversos niveles y su posición respecto a los polos frontal y occipital y al margen superior del hemisferio cerebral.

\section{MATERIAL Y MÉTODO}

Se utilizaron 20 cerebros frescos, de los cuales se expuso la cara medial de los hemisferios, donde se localizó

\footnotetext{
* Facultad de Medicina, Universidad de La Frontera, Chile.

** Universidad Mayor, Sede Temuco, Chile.

**** Universidad Autónoma de Chile, Sede Talca, Chile.
} 
al cuerpo calloso, fotografiando esta estructura y a las partes vecinas del hemisferio. Antes de obtener la fotografía, cada una de las muestras fueron acompañadas con una regla, con el objetivo de trabajar sobre la imagen y de este modo registrar las variables planificadas.

Las siguientes variables fueron determinadas:

- Distancia entre polos frontal y occipital.

- Distancia entre la parte más sobresaliente de la rodilla y el margen anterior del hemisferio cerebral.

- Distancia entre la parte más prominente del esplenio y el margen posterior del hemisferio cerebral.

- Distancia entre el margen superior del cuerpo calloso y el margen superior del hemisferio cerebral.

- Longitud antero-posterior del cuerpo calloso.

- Distancia antero-posterior de la parte media de la rodilla.

- Distancia antero-posterior de la parte media del esplenio.

- Espesor de la parte media de: el cuerpo calloso, de la rodilla y del esplenio.

De cada uno de estos parámetros se obtuvo promedio y desviación standard, se esquematizó la estructura en estudio y se fotografiaron cada una de sus partes.

\section{RESULTADOS}

El promedio de la distancia entre los polos frontal y occipital fue de $188,5 \mathrm{~mm} \pm 9,6$; la distancia promedio obtenida entre la parte más prominente de la rodilla del cuerpo calloso y el margen anterior del hemisferio cerebral fue de $35,4 \pm$ $5,1 \mathrm{~mm}$ y entre la parte más prominente del esplenio y el margen posterior del hemisferio cerebral fue de $52,8 \mathrm{~mm} \pm 5,4$.

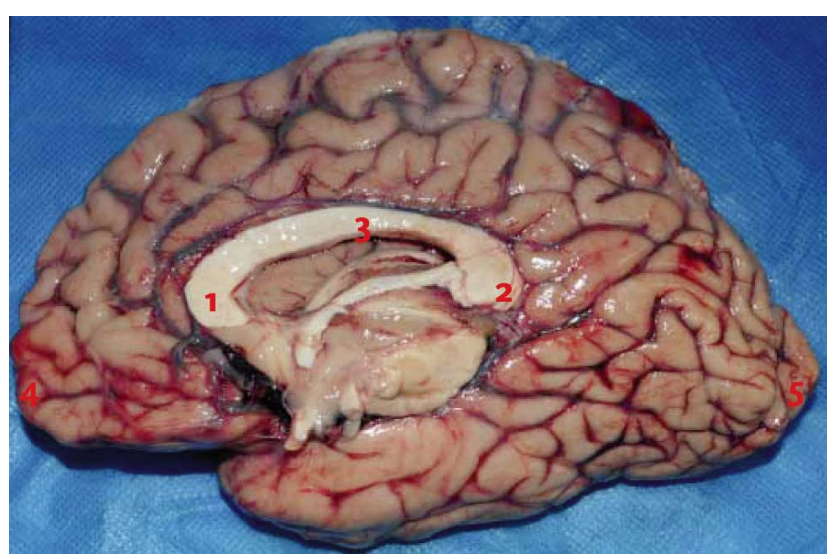

Fig.1.- Hemisferio derecho, 1. Rodilla cuerpo calloso; 2. Esplenio del cuerpo calloso; 3 . Tronco del cuerpo calloso; 4. Polo anterior del hemisferio; 5. Polo posterior del hemisferio.

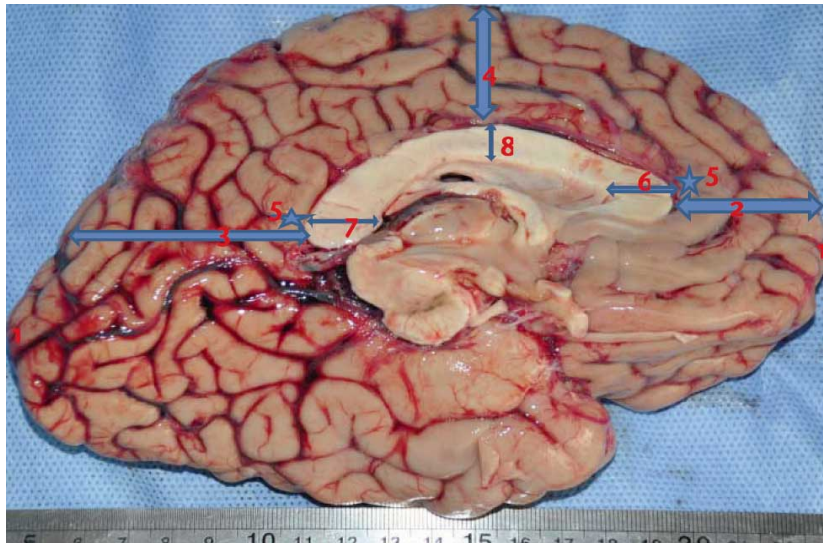

Fig.2.- 1-1'. Distancia entre los polos occipital y frontal; 2. Distancia rodilla-margen anterior lóbulo frontal, 3. Distancia esplenio-margen posterior del lóbulo occipital; 4. Distancia cara superior tronco cuerpo calloso-margen superior hemisferio; 5. Longitud A-P del cuerpo calloso (entre estrellas); 6. Espesor A-P rodilla, 7; Espesor A-P esplenio; 8. Espesor tronco.

La longitud antero-posterior del cuerpo calloso fue en promedio $88,2 \mathrm{~mm} \pm 7,6$; la distancia antero-posterior promedio de la parte media de la rodilla fue $13,2 \mathrm{~mm} \pm 2,3$ y la de la parte media del esplenio $15,7 \mathrm{~mm} \pm 2,1$; su espesor promedio medido a nivel del punto medio de su longitud fue de $8,7 \mathrm{~mm} \pm 1,5$; el espesor en la parte media de la rodilla fue de $14,2 \mathrm{~mm} \pm 2,2$ y en la parte media del esplenio fue de $17,0 \mathrm{~mm} \pm 2,6$ (Figs.1 y 2).

La distancia entre la parte superior de la zona media del cuerpo calloso al margen superior del hemisferio cerebral fue de $32,9 \mathrm{~mm} \pm 6,5$.

\section{DISCUSIÓN}

Varios autores han investigado el cuerpo calloso, estructura comisural de sustancia blanca, sobre sus características morfológicas, intentando comparar entre los individuos el espesor del esplenio, principalmente en lo que se refiere a dimorfismo sexual (Allen et al.; Bishop \& Wahlsten; Dorion et al. y Battal et al.). No realizamos comparaciones entre sexos, ya que la mayor parte de las muestras utilizadas en este estudio pertenecían a individuos de sexo masculino.

Las fibras comisurales frontales cruzan en el rostrum del cuerpo calloso; las fibras comisurales parietales cruzan en el tronco y las fibras comisurales occipitales cruzan en el esplenio. Normalmente, ayuda a unir las actividades de los dos hemisferios cerebrales. Una sección de fibras del cuer- 
po calloso puede disociar los dos hemisferios y literalmente, producir un individuo con dos cerebros distintos. Anormalidades derivadas del desarrollo pueden llevar a una agenesia parcial o total del cuerpo calloso. La agenesia ocurre entre 1 y 3 de cada 1000 nacimientos y es más predominante en niños con malformaciones cerebrales adicionales o con enfermedades metabólicas (Willard, 1993).

También, se ha calculado el número aproximado de fibras que interconectan los hemisferios y que pasan a través del cuerpo calloso (Aboitiz et al., 1992), informándose que éste posee $1,6 \times 10^{8}$ axones. Estos autores indicaron que las fibras de menor calibre (mayor densidad) se encuentran en la rodilla y en el sector del esplenio, pero en el polo posterior del cuerpo calloso la densidad decrece. Las fibras más gruesas se encuentran en la parte posterior de la zona central del tronco. También señalaron que no hubo diferencias entre sexos, en la composición de las fibras.

Estudios sobre las características biométricas de esta importante estructura son escasos, de tal modo que los datos entregados por esta investigación en cerebros frescos de nuestra población son un aporte al conocimiento de ésta. Estudios sobre su morfología, ya sea a través de anatomía directa o a través de tomografías, han sido realizados por Allen et al.; Bishop \& Wahlsten; Dorion et al.; Battal et al., autores ya mencionados.

En la mayoría de los textos se describe que el cuerpo calloso tiene una longitud de aproximadamente $10 \mathrm{~cm}$, valor que en este estudio ha resultado un poco menor, con un promedio de $8,8 \mathrm{~cm}$. Con respecto al espesor del mismo, fue posible apreciar que la parte media del esplenio fue mayor que en la rodilla y que ambos valores fueron mayores que en la parte media del cuerpo.

La distancia entre la parte superior del tronco del cuerpo calloso respecto al margen superior del hemisferio cerebral, es semejante a la distancia entre la parte más prominente del la rodilla respecto al margen frontal, no así la distancia entre la parte más prominente del esplenio y el margen occipital, la cual fue mayor y en promedio equivale a una relación aproximada de 1,5:1,0.

Los resultados obtenidos constituyen un aporte a la anatomía del cuerpo calloso en nuestra población, complementando el conocimiento biométrico sobre esta importante comisura.

OLAVE, E.; TORREZ, J. C.; RIQUELME, N. ; IBACACHE, L. \& BINVIGNAT, O. Biometric characteristics of the corpus callosum in Chilean subjects. Int. J. Morphol., 30(4):1449-14528, 2012.

SUMMARY: The corpus callosum is a structure of white matter connecting the two cerebral hemispheres and is located at the bottom of the longitudinal fissure of the brain. In order to complement the understanding of this important structure of the nervous system in our population, we determined various biometric parameters, which included its location in relation to the anterior and posterior margins of the cerebral hemispheres, length, thickness at various levels, among other variables. To do this, we used 20 fresh brains of adult individuals which the medial aspect of the hemispheres was photographed, and were accompanied with a ruler before you get the picture, with the aim of working on the image and thus record the planned variables. The average distance obtained between the most prominent part of the knee of the corpus callosum and the anterior margin of the cerebral hemisphere was $35.4 \mathrm{~mm}$ and among the most prominent part of the splenium and the posterior margin of cerebral hemisphere was $52.8 \mathrm{~mm}$. Anteroposterior length of the corpus callosum was on average $88.2 \mathrm{~mm}$, the average antero-posterior diameter from the middle of the knee was $13.2 \mathrm{~mm}$ and that of the middle part of splenius $15.7 \mathrm{~mm}$. The data obtained contribute to the biometric and morphological knowledge of this important commissural structure in our population group.

KEY WORDS: Anatomy; Nervous System; Comissures; Corpus callosum; Biometry.

\section{REFERENCIAS BIBLIOGRÁFICAS}

Aboitiz, F.; Scheibel, A.B.; Fisher, R.S. \& Zaidel, E. Fiber composition of the human corpus callosum. Brain Res., 598(12):143-53, 1992.

Allen, L.S.; Richey, M.F.; Chai, Y.M. \& Gorski, R.A. Sex differences in the corpus callosum of the living human being. J. Neurosci., 11(4):933-42, 1991.
Battal, B.; Kocaoglu, M.; Akgun, V.; Bulakbasi, N. \& Tayfun, C. Corpus callosum: Normal imaging appearance, variants and pathologic conditions. J. Med. Imaging Rad.Oncol., 54:541-549, 2010.

Bishop, K.M. \& Wahlsten, D. Sex differences in the human corpus callosum: myth or reality? Neurosci. Biobehav. Rev., 21(5):581601, 1997. 
OLAVE, E.; TORREZ, J. C.; RIQUELME, N. ; IBACACHE, L. \& BINVIGNAT, O. Características biométricas del cuerpo calloso en individuos chilenos. Int. J. Morphol., 30(4):1449-1452, 2012.

Dorion, A.A.; Capron, C. \& Duyme, M. Measurement of the corpus callosum using magnetic resonance imaging: analyses of methods and techniques. Percept. Mot. Skills, 92(3 pt2):107594, 2001.

Snell, R. Neuroanatomía Clínica. $5^{\mathrm{a}}$ Ed. Buenos Aires, Panamericana, 2001.

Willard, F.H. Medical Neuroanatomy. Philadelphia, J. B. Lippincott Company, 1993.

Williams, R L.; Warwick, R.; Dyson, M. \& Bannister, L. H. Gray Anatomía. 37 ${ }^{\text {a }}$ Ed. Rio de Janeiro, Guanabara-Koogan, 1995. V. 2.
Dirección para correspondencia:

Prof. Dr. Enrique Olave

Facultad de Medicina

Universidad de La Frontera

Temuco

CHILE

Email: eolave@ufro.cl

Recibido : 22-04-2012

Aceptado: 18-06-2012 